\title{
Efficacy of multi-groove silicone drains in single-port video-assisted thoracoscopic lung cancer surgery and their effect on C-reactive protein: a single-center experience
}

\author{
Yuanshan Yao ${ }^{1}$, Qingwang $\mathrm{Hua}^{2}$, Suyue $\mathrm{Liu}^{2}$, Zhenhua Yang ${ }^{2}$, Haibo Shen ${ }^{2}$, Wen Gao ${ }^{1}$ \\ ${ }^{1}$ Department of Thoracic Oncology, Shanghai Key Laboratory of Clinical Geriatric Medicine, Huadong Hospital Affiliated to Fudan University, \\ Shanghai, China; ${ }^{2}$ Department of Thoracic Oncology, Ningbo No. 2 Hospital, Ningbo, China \\ Contributions: (I) Conception and design: Y Yao; (II) Administrative support: W Gao; (III) Provision of study materials or patients: H Shen; (IV) \\ Collection and assembly of data: Y Yao, Q Hua, S Liu, Z Yang; (V) Data analysis and interpretation: Y Yao, W Gao; (VI) Manuscript writing: All \\ authors; (VII) Final approval of manuscript: All authors. \\ Correspondence to: Dr. Wen Gao. Department of Thoracic Oncology, Shanghai Key Laboratory of Clinical Geriatric Medicine, Huadong Hospital \\ Affiliated to Fudan University, Shanghai 200041, China. Email: gaowenchest@163.com.
}

Background: The purpose of this study was to systematically evaluate the effectiveness and safety of a multi-groove silicone drain in single-port video-assisted thoracoscopic lung cancer surgery and its effect on postoperative serum C-reactive protein (CRP) levels.

Methods: We retrospectively analyzed 122 surgical cases who underwent standard lobectomy and lymph node dissection for primary lung cancer between May 2020 and December 2020. A total of 62 patients received 19-F multi-groove silicone drains (experimental group) and 60 patients received 24-F conventional chest drains (control group). According to the different thoracic drainage approaches, the clinical efficacy in the perioperative period, postoperative complications, and postoperative serum CRP levels were compared between the 2 groups.

Results: In this study, thoracic drainage volume, the average visual analog scale (VAS) pain scores in incisions, the rate of primary healing at the site of incisions, and the pulmonary infection rate in the multigroove silicone drain group were significantly lower than those in the conventional chest drain group $(\mathrm{P}<0.05)$, but there was no significant difference in the average hospital stay time, arrhythmia rates, and chest tube removal time between the 2 groups. At postoperative day 1, the levels of serum CRP in the 2 groups were further increased $(\mathrm{P}>0.05)$, and the comparison between the 2 groups showed that the levels of serum CRP in the multi-groove silicone drain group at $72 \mathrm{~h}$ after the operation were significantly lower than those in the conventional drain group $(\mathrm{P}<0.05)$.

Conclusions: Our results showed that a multi-groove silicone drain is feasible and relatively safe in singleport video-assisted thoracoscopic lung cancer surgery for most patients. However we should take cautious in those patients with higher susceptibility of postoperative active bleeding. In patients undergoing lung cancer surgery in the clinical treatment process, the use of a multi-groove silicone drain can improve the quality of life of patients. Due to a small number of included studies and unclear bias, the above results should be verified by high-quality, large-sample randomized controlled studies.

Keywords: Video-assisted thoracoscopic lung cancer surgery; multi-groove silicone drains; conventional chest drains

Submitted Oct 26, 2021. Accepted for publication Dec 15, 2021.

doi: $10.21037 /$ jtd-21-1801

View this article at: https://dx.doi.org/10.21037/jtd-21-1801 


\section{Introduction}

Lung cancer is one of the most common types of malignant tumor, with a sharply increasing incidence and mortality rate. Worldwide, lung cancer has become the leading cause of death among other cancers, and its mortality rate has increased $65.23 \%$ compared with that in the 1990 s (1). In recent years, with the significant development of lowdose helical computed tomography (CT), the percentage of stage I and stage II lung cancers was among the highest in all lung cancer cases after lung screen trial (2). Along with the improvement of thoracoscopic surgery techniques and equipment, single-port video-assisted thoracoscopic surgery for the radical treatment of lung cancer has gained considerable attention from thoracic surgeons. In contrast with multiports surgery, single port surgery is considered as less postoperative pain involved, less intercostal nerves injury and more exciting outcome. Additionally, the good results of enhanced recovery after surgery (ERAS) combined with single-port video-assisted thoracoscopic surgery are firmly believed to have contributed to lowering the incidence of perioperative complications and shortening the length of chest tube removal time $(3,4)$. The chest tube, as one of the most important elements in the ERAS program after lung cancer surgery, has attracted growing attention from clinicians. Drainage is established with negative intrapleural pressure through constantly draining air and fluids from the chest cavity to allow a full expansion of the residual lung. Owing to the large diameter and relative rigidity of these tubes, patients often experience pain at the entry site through the skin and muscles, as well as between the ribs. At the same time, conventional chest drains have some inherent drawbacks, such as poor wound healing, pain in incisions that can limit ambulation and deep breathing, and leakage of fluids or air around the drainage orifice, among other issues (5). Omitting the chest tube was tested in several selected patients, however, due to the drawback of postoperative thoracic bleeding could not be observed, this procedure needs to be validated in large cohort (6). As a result, all of these problems can delay postoperative recovery. Hence, evaluating the chest tube used in single-port video-assisted thoracoscopic lung cancer surgery has received increasing attention. A recent study by Wang etc. using a multi-groove silicone drain combined with negative-pressure drainage system after lung surgery has been reported (7). He showed his system can replace the conventional chest tube. We undertook a study to analyze almost the same multi-groove silicone drain combined with ordinary thoracic drainage bottle in lung surgery and found that this multi-groove silicone drain seems to be effective and can be used as an alternative in most patients, however this drain can be obstructed in extreme cases.

In recent years, foreign researchers have explored the relationship between postoperative serum C-reactive protein (CRP) level and complications after surgery $(8,9)$. It is commonly believed that CRP can act as an acute-phase response protein (10). At present, some scientific inquiries have already proven the close relationship between CRP and the progression and development of malignancies (11-13). Now CRP is pervasively used in multiple aspects of lung cancer patients. Hence, we presumed that CRP may be correlated with different thoracic drains approach.

The purpose of this study was to evaluate our experience with the use of small and soft $19 \mathrm{~F}$ multi-groove silicone drains in single-port video-assisted thoracoscopic lung cancer surgery and its effect on CRP levels.

We present the following article in accordance with the STROBE reporting checklist (available at https://dx.doi. org/10.21037/jtd-21-1801).

\section{Methods}

\section{General materials}

Patients who underwent single-port video-assisted thoracoscopic lung cancer surgery in our hospital from July 1, 2020 to December 31, 2020 were retrospectively analyzed. According to the Chinese Medical Association guidelines for the clinical diagnosis and treatment of lung cancer (edition 2018), all patients underwent a standard lobectomy and mediastinal lymph node dissection. The inclusion criteria were as follows: (I) All patients underwent preoperative chest enhanced CT for the confirmation of the clinical stage, and all patients were pathologically considered as lung cancer in intraoperative frozen section analysis which was performed by an expert in thoracic surgery; (II) patients aged 18-75 years old; (III) reserves and compensatory capacity of the heart, lung, liver, and kidneys of all the patients were carefully evaluated before the operation, and all patients were confirmed to tolerate lung cancer operations; (IV) patients who did not smoke cigarettes in the past 2 weeks; (V) patients whose coagulation was normal and those that did not take anticoagulants in the past 2 weeks. The study was conducted in accordance with the Declaration of Helsinki (as revised in 2013). The study was approved by ethics board of 


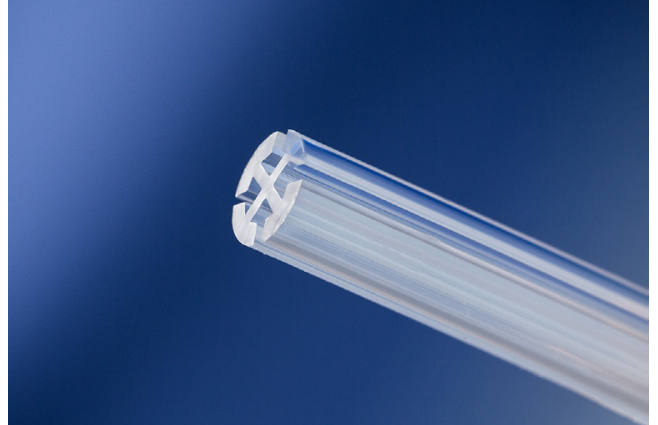

Figure 1 Multi-groove silicone drain.

Ningbo No. 2 Hospital (No.: 2021-047-01), and individual consent for this retrospective analysis was obtained.

\section{Study methods}

\section{Multi-groove silicone drain group}

After adequate preparations, surgical treatment was conducted. Patients were placed in the lateral position and generally received one-lung ventilation. The single port method was applied: a single, muscle-sparing 3 to $4 \mathrm{~cm}$ incision was made through the 4th or 5 th intercostal space according to different heights and somatotype characteristics. No other incision was made. All of the instruments and camera were inserted through this incision. At the end of lobectomy and lymphadenectomy, a multigroove silicone drain was inserted through the incision (14). The multi-groove silicone drain is $\mathrm{X}$-ray opaque and made of medical grade silicone equipped with 4 longitudinal grooves so that tissues and thrombus do not completely occlude the grooves. The multi-groove silicone drain we used is manufactured by "Ai Ze" corporation, China (Figure 1). The drains used in this study were $19 \mathrm{Fr}$ and $6.3 \mathrm{~mm}$ in diameter. All of the chest drains were inserted from the incisions straight to the top of the chest through the anterior mediastinum pathway. All of the drains were connected to water-seal bottles without negative pressure, and analgesia pumps were not routinely used after the operation.

Postoperative bedside chest X-rays are shown in Figure 2. Postoperatively, the patients were treated by routine ERAS procedures. Drainage volume was recorded the morning after the operation day by nurses. On the basis of visual analog scale (VAS) score, the average pain scores in incisions were recorded by 2 experienced nurses, and the averages for postoperative day 1 and discharge day were calculated (15). Lung re-expansion, subcutaneous emphysema, and

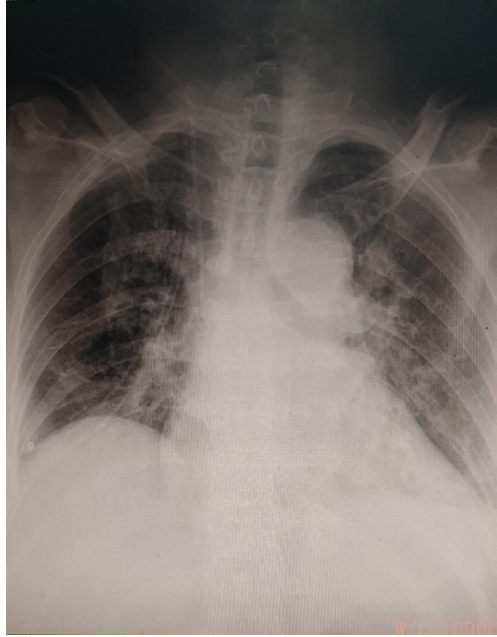

Figure 2 Multi-groove silicone drain in chest cavity in bedside $\mathrm{X}$-ray.

residual effusion in the chest after chest tube removal were confirmed by chest X-ray or chest CT. Postoperative pulmonary infections were defined as follows: high temperature above $38.5^{\circ} \mathrm{C}$; persistent symptoms such as cough, expectoration, and gasp; some pulmonary infiltration inflammation in postoperative chest X-ray or chest CT (16). We recorded complications after surgery such as pulmonary complications, arrhythmia rates, and the bleeding situation in the perioperative period.

\section{Conventional chest drain group}

Radical surgery for lung cancer was completed by the same thoracic surgeon. At the end of surgery, we use a conventional chest drain as an alternative. The conventional chest drains used were $24 \mathrm{Fr}$ and $8 \mathrm{~mm}$ in diameter. The postoperative recovery plan were the same as the experimental group.

\section{Chest tube removal and discharge criteria}

The removal of chest tubes was standardized by protocol after lung resection. Patients with no air leakage lasting for $24 \mathrm{~h}$ and total light yellow fluid drainage $<400 \mathrm{~mL}$ per $24 \mathrm{~h}$ were considered for chest tube removal. X-ray or chest CT showing residual lung re-expansion and no obvious pneumonitis, along with no patient complaints were acceptable criteria for discharge $(7,17)$.

\section{Observation indexes}

Postoperative thoracic drainage volume, arrhythmia rates, 
the average VAS pain scores in incisions, the rate of primary healing at the site of incisions, average hospital stay time, pulmonary complications, and chest tube removal time during the perioperative period were collected. In addition, levels of serum CRP in the 2 groups of patients before the operation, $24 \mathrm{~h}$ after operation, and $72 \mathrm{~h}$ after the operation were also included.

\section{Statistical methods}

Statistical Product and Service Solutions (SPSS) 26.0 software was used to analyze the data. The measurement data were expressed as mean \pm standard deviation $(\bar{x} \pm s)$, and the comparison between the 2 groups was performed using the $t$-test. The count data was expressed as a percentage (\%), and the $\chi^{2}$ test was used for comparisons. Fisher's exact test was used to calculate the p-value if the chi-square test was not suitable. The inspection level was $\alpha=0.05$.

\section{Results}

\section{Clinical characteristics}

A total of 122 patients were enrolled in the study. Patients were classified into the multi-groove silicone drain group $(n=62)$ and the conventional chest drain group $(n=60)$ according to the different drainage methods. The baseline characteristics of the 2 groups were compared and no significant differences were found ( $\mathrm{P}>0.05$; Table 1).

\section{Intraoperative situation}

All of the patients underwent successful surgery. With regard to blood loss, average operation time, and rate of pleural adhesion, a comparison of both groups revealed that there were no differences (Table 2).

\section{Postoperative situation}

\section{Postoperative recovery situation}

The thoracic drainage volume and the average VAS pain scores in incisions in the multi-groove silicone drain group were significantly lower than those in the conventional chest drain group $(\mathrm{P}<0.05)$. The average hospital stay time and chest tube removal time showed no differences between the 2 groups $(\mathrm{P}>0.05$; Table 3). Meanwhile, we compared the variations in the amount of postoperative drainage volume over time between the 2 different kinds of drains in patients after lobectomy and lymph node dissection for primary lung cancer. Postoperative day 1-5 drainage volumes were retrospectively compared between two groups, respectively (Figure 3).

\section{Postoperative complications}

Our study showed that about one third of patients experience postoperative complications in our groups. Early complications usually consist of atelectasis, pneumonia, prolonged air leakage, pulmonary edema, acute respiratory distress syndrome, arrhythmia, and bleeding. Late complications mainly include bronchopleural fistula, wound infection, and empyema (18). Due to adequate preoperative preparations and intraoperative care such as pulmonary rehabilitation, smoking cessation, aerosol inhalation, correction of electrolyte disorders, and intensive nutritional support, life-threating complications including acute respiratory distress syndrome, malignant ventricular arrhythmia, empyema, and bronchopleural fistula did not occur. The pulmonary infection rate and the rate of primary healing at the site of incisions in the multi-groove silicone drain group were lower than those in the conventional chest drain group $(\mathrm{P}<0.05)$. However, there were significant differences in terms of arrhythmia and overall pulmonary complications between the 2 groups (Table 4). Among all patients, through only observation or the use of antiarrhythmic drugs, 19 patients with arrhythmia recovered. There were 2 patients with atelectasis after bedside X-ray who improved through active inhalation drug therapy, passive turn over and patting of the body, or bronchoscopy suction. There were 24 patients with pulmonary infection which was cured by vibratory therapy, allowing patients to clear their own secretions, and anti-infection treatments. There were 9 patients with air leakage 5 days after the operation, which could be stopped by intrapleural injection of hypertonic glucose solution or iodophor diluent after the residual lung fully re-expanded. There was only 1 patient with thoracic hemorrhage in the multi-groove silicone drain group who was treated with a small 8-F Abel drainage tube in the subsequent 2 weeks after discharge without being treated with repeated thoracotomy. There were no patients with fat liquefaction, wound infection, and deep venous thrombosis due to obesity or uncontrolled diabetic blood sugar.

\section{Changes in serum CRP level after surgery}

The level of serum CRP after surgery was higher than that 
Table 1 Comparison of clinical data of patients between the multi-groove silicone drain and conventional drain groups

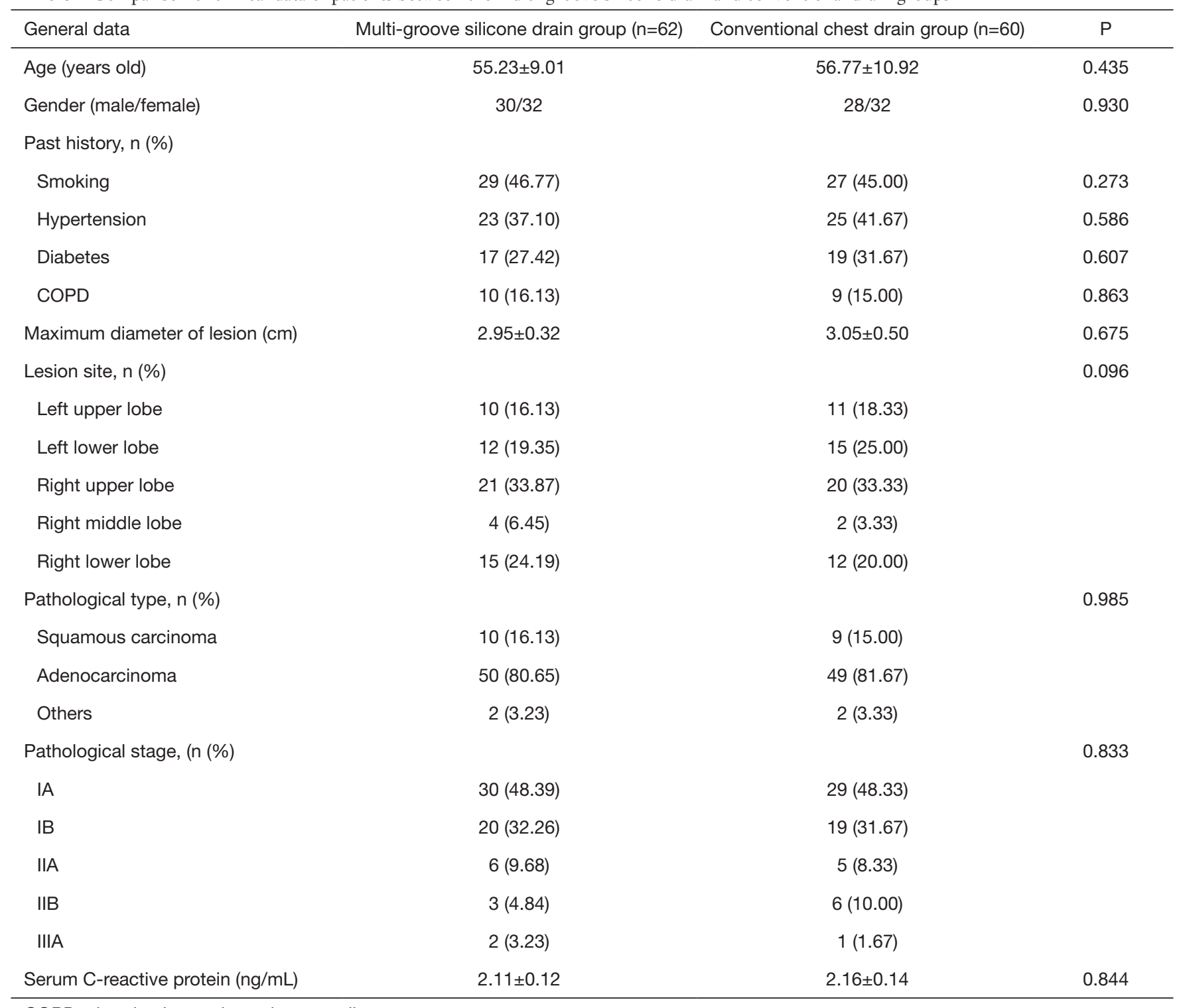

COPD, chronic obstructive pulmonary disease.

Table 2 Comparison of the intraoperative situation of patients between the 2 groups

\begin{tabular}{|c|c|c|c|}
\hline Intraoperative situation & Multi-groove silicone drain group $(n=62)$ & Conventional chest drain group $(n=60)$ & $\mathrm{P}$ \\
\hline Intraoperative blood loss (mL) & $150.23 \pm 45.22$ & $158.54 \pm 32.45$ & 0.417 \\
\hline Pleural adhesion, $\mathrm{n}(\%)$ & $6(9.68)$ & $5(8.33)$ & 0.124 \\
\hline
\end{tabular}


Table 3 Comparison of postoperative recovery situation between the 2 groups

\begin{tabular}{|c|c|c|c|}
\hline Postoperative indexes & Multi-groove silicone drain group $(n=62)$ & Conventional chest drain group $(n=60)$ & $P$ \\
\hline Thoracic drainage volume (mL) & $624.57 \pm 231.15$ & $1045.78 \pm 156.95$ & 0.030 \\
\hline Average VAS pain scores in incisions (score) & $1.10 \pm 0.50$ & $4.20 \pm 0.70$ & 0.010 \\
\hline Average hospital stay time (h) & $6.58 \pm 1.98$ & $6.67 \pm 2.07$ & 0.214 \\
\hline
\end{tabular}

VAS, visual analog scale.

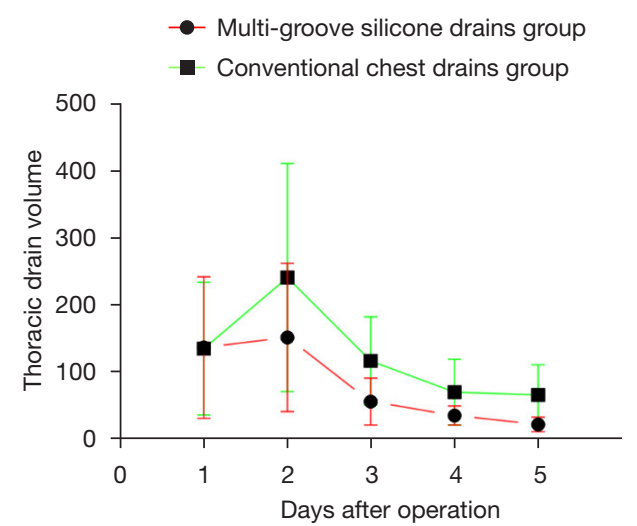

Figure 3 Comparison of thoracic drain volume after operation between the 2 groups. before surgery, and at $24 \mathrm{~h}$ after surgery the amount of serum CRP in the 2 groups further increased $(\mathrm{P}>0.05)$. The comparison between the 2 groups showed that the levels of serum CRP in the multi-groove silicone drain group at $72 \mathrm{~h}$ after surgery were significantly lower than those in the conventional drain group $(\mathrm{P}<0.05$; Table 5 and Figure 4).

\section{Discussion}

Video-assisted thoracoscopic surgery has been extensively used in the treatment of various benign and malignant pulmonary diseases since the 1990s $(19,20)$. Along with the significant development of thoracoscopic surgery skills, techniques, and equipment, single-port video-assisted

Table 4 Comparison of postoperative complications of patients between the 2 groups

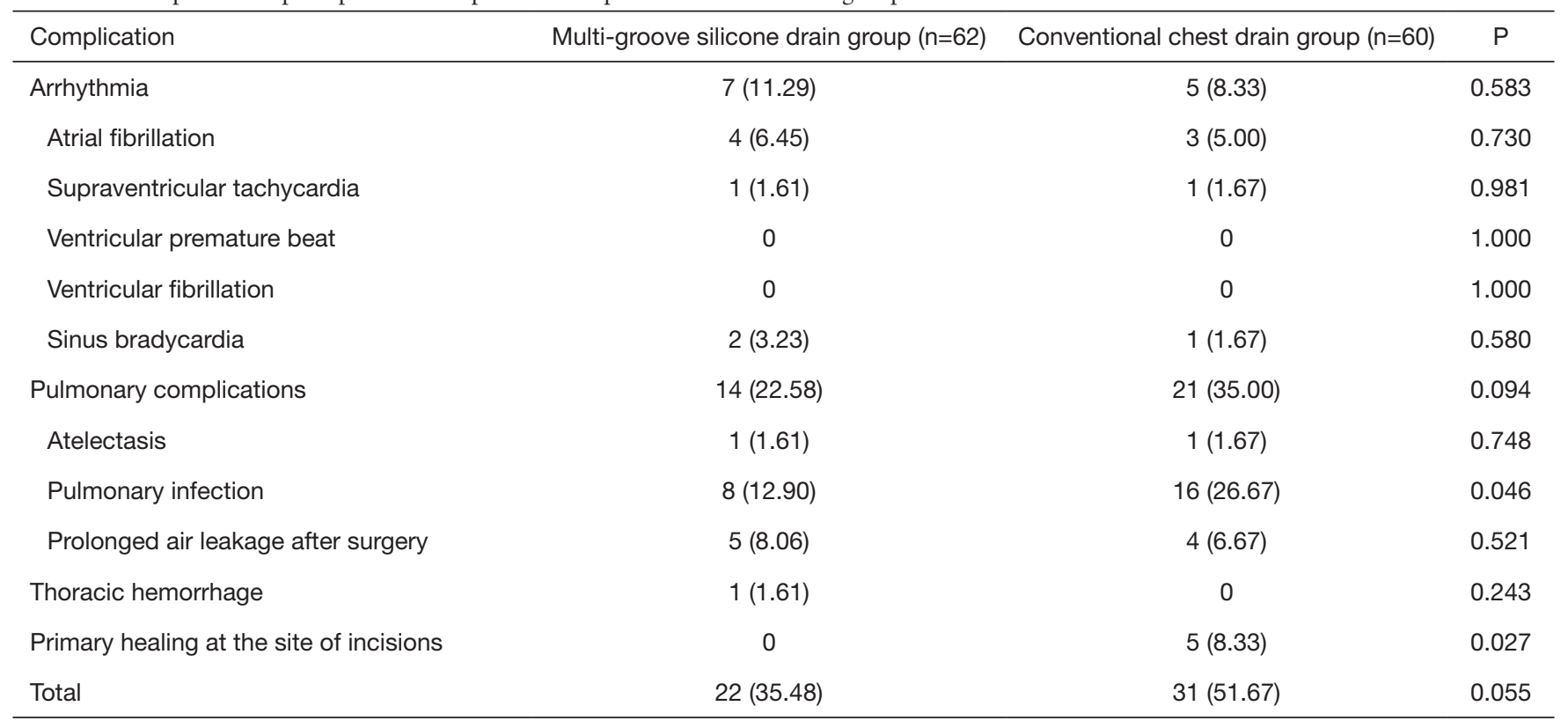

Data presented as $\mathrm{n}(\%)$. 
Table 5 Comparison of changes in the C-reactive protein levels of patients after surgery between the 2 groups

\begin{tabular}{|c|c|c|c|}
\hline Serum C-reactive protein & Multi-groove silicone drain group $(n=62)$ & Conventional chest drain group $(n=60)$ & $P$ \\
\hline At $24 \mathrm{~h}$ after operation, $(\mathrm{ng} / \mathrm{mL})$ & $120.7 \pm 4.1$ & $140.2 \pm 6.9$ & 0.098 \\
\hline At $72 \mathrm{~h}$ after operation, $(\mathrm{ng} / \mathrm{mL})$ & $20.9 \pm 5.2$ & $85.4 \pm 6.2$ & 0.015 \\
\hline
\end{tabular}

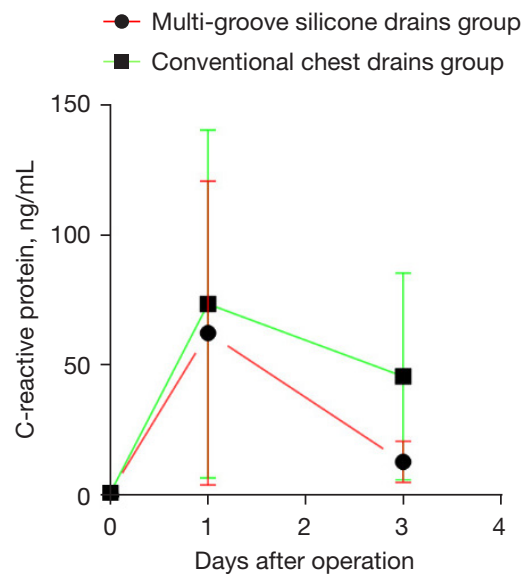

Figure 4 Comparison of C-reactive protein after operation between the 2 groups.

thoracoscopic surgery is increasingly becoming the first choice for the radical treatment of lung cancer and has gained considerable attention from thoracic surgeons. Compared to the multiple ports approach, 1 intercostal incision is used to perform the surgery, which theoretically lessens the destruction to the intercostal nerves and causes less pain (21). In recent years, the single-port VATS approach has also extended to different kinds of thoracic surgical procedures, such as wedge resection, lobectomy, segmentectomy, minimally invasive esophagectomy, and the excision of mediastinal masses (22). Some studies have clearly confirmed that single-port video-assisted thoracoscopic lobectomy is an acceptable procedure with a low morbidity and mortality rate (23). However, Borro et al. in 2017 doubted that single-port VATS was correlated with a higher risk of death and the appearance of tumor local recurrence and metastasis in their retrospective review of 276 VATS lobectomies (24). To our knowledge, the increased difficulty of some operative instruments combined with a thoracoscopic camera in a small incision, prolonged operation time, and perhaps some difficulties in handling the intraoperative complications may be responsible for this situation.

Some skilled thoracic surgeons had to relearn disparities between three ports and one port surgery in a short time. With the adaptation to these new surgical skills, surgeons will perform the surgery more proficiently. Now, singleport video-assisted thoracoscopic lung cancer surgery is becoming a routine surgery not only performed in a limited number of hospitals but also some municipal hospitals $(25,26)$. We recommended there were several ways to develop single-port video-assisted thoracoscopic lung cancer surgery skills. Firstly, learning to use the surgical instruments by left hand and this behavior will make us more proficiently. Secondly, using a simulated box trainer at home will promote us to learn the skills quickly instead of learning in the operation room.

Anesthesia, nursing care, and surgery are core concepts in ERAS, which is becoming increasingly acceptable to most thoracic surgeons. Postoperative pain relief is indispensable in enhancing the rapid recovery of patients. Postoperative pain may arise from 3 aspects: (I) intercostal nerve compression or damage by chest tubes; (II) chest tube stimulating the diaphragm or pleura; (III) mental stress. Therefore, chest tubes play a crucial role in ERAS.

Traditionally, chest tubes are used to drain both air and fluids through establishing negative pressure in the chest. Safe and effective chest tubes are indispensable to prevent tension pneumothorax, identify latent hemorrhage, and to reduce asymptomatic pleural fluids. In the past 10 years, we have usually inserted two 28 - or $34-\mathrm{F}$ chest tubes in the chest cavity at the end of the operation. One 34-F chest tube, which we often called the top chest tube, was directly advanced to the apex of the chest cavity for the sake of draining air smoothly, while the other 28- or 34-F chest tubes, which we often called below chest tubes, were advanced to the lowest point of the thoracic cavity in order to drain fluids $(7,17,27)$. We have used large diameter chest tubes instead of relatively smaller chest tubes in the past few years, mainly because once a large amount of pulmonary alveolar or small bronchiolar fistulas appeared, we were concerned smaller chest tubes would not offer adequate 
air drainage that exceeded the speed of pulmonary alveolar fistula, thus air could penetrate the muscles of the wound and subsequently form subcutaneous emphysema (28). The majority of cases of subcutaneous emphysema can resolve on their own, though it can cause panic and discomfort among these patients, therefore prolonging their hospital time (29,30). Nevertheless, conventional chest tubes can also lead to several problems, such as intercostal nerve pain, poor incision healing, and high thoracic drainage volume that can potentially cause massive nutritional loss.

Multi-groove silicone drains, which are often called Blake drains in foreign countries, have been extensively used for thoracic drainage after cardiac surgeries in both pediatric and adult patients from 2001 (31). However, they have not been commonly used for thoracic drainage in lung cancer surgeries in recent years, mainly because thoracic surgeons did not believe this chest drain could efficiently drain air and fluids from the residual space due to lung resection, so their advantages are still unclear. The multi-groove silicone drain is used for large area drainage and is flexible, made of medical grade silicone equipped with 4 longitudinal grooves so that tissues cannot entirely obstruct the grooves (32). Our institution has used multi-groove silicone drainage instead of conventional 24-F chest tubes for most general thoracic surgeries performed since 2020 and has achieved excellent results.

Wang et al. demonstrated that thoracic drainage volume and poor incision healing in the multi-groove silicone drain group were lower than in the thoracic tube group (7). In our study, thoracic drainage volume, the average VAS pain scores in incisions, the rate of primary healing at the site of incisions, and the pulmonary infection rate in the multi-groove silicone drain group were lower than those in the conventional chest drain group $(\mathrm{P}<0.05)$. Because of their soft texture and strong flexibility, multi-groove silicone drains can alleviate pain accompanied with intercostal nerve compression, increase the rate of primary healing at the site of incisions caused by soft tissue extrusion by the drain, and further lessen the psychological, economic burden, and impaired social function of patients due to wound healing (33). Besides, total thoracic drainage volume in the multi-groove silicone drain group was lower than the conventional chest drain group $(\mathrm{P}=0.030)$. We postulate that soft texture multigroove silicone drains in the chest can stimulate less fluids and quickly promote residual lung re-expansion so that there exists no space to generate fluids. More interestingly, in Figure 3, we can see that at postoperative day 1 the thoracic drainage volume in the 2 groups after the operation was almost the same, and the thoracic drainage volume was further increased at $48 \mathrm{~h}$ after the operation and reached the peak simultaneously. After postoperative day 2, the thoracic drainage volume begun to decrease. The thoracic drainage volume output mainly consists of vascular exudations, pleural effusions due to chest tube stimulation and impairment of mesothelial cells of the pleura after surgery, and lymphatic fluid due to mediastinal lymph node dissection $(34,35)$. At postoperative day 2 , we usually supply patients with a general diet compared with a semi-liquid diet at postoperative day 1 . Therefore, lymphatic fluid will increase with a fatty diet. However, we did not encounter chylothorax mainly because we used hemo-lock clips in the mediastinal lymph node dissection field. Then, in accordance with lower vascular exudations and pleural fluid recycling, the thoracic drainage volume will decrease after postoperative day 2. The multi-groove silicone drains not only enhanced the degree of satisfaction but also brought less pain and reduced the level of discomfort of patients during changing the gauze around the wound or removing the drains. The multi-groove silicone drains are believed to be less painful for patients, enabling earlier mobilization after surgery and in the meantime inducing patients to cough strongly and expel sputum gathered in the airway after surgery, therefore reducing the chance of pulmonary infection $(28,36)$. This is in contrast to the conventionally used large sized chest drains, which may hinder the postoperative recovery by limiting ambulation and interfering with effective chest physiotherapy $(37,38)$. There was no significant difference in the average hospital stay time, arrhythmia rates, and chest tube removal time between the 2 groups. In addition, the multi-groove silicone drainage group had a similar percentage of prolonged air leak than those in the conventional chest tube drainage group. The efficacy of air evacuation is considered to be the predominant aspect of chest tube performance after pulmonary surgery. Sakakura et al. displayed that once prolonged air leak happened, suction were necessary tools (36). Suction might help residual lung re-expansion as soon as possible, This result is similar to that in the study of Sakakura et al.

Unfortunately, in the multi-groove silicone drain group, there was a case of left lower lobe resection complicated by postoperative intrathoracic bleeding that was not suspected until the onset of hypotension. Useful chest drainage should promote recognition of ongoing bleeding quickly because of red blood effusion. However, we did not become aware of this situation until hypotension and then bedside $\mathrm{X}$-ray had confirmed this complication. No minimal 
amounts of blood were observed from the thoracic cavity in the drain. We immediately administered fluid resuscitation and prepared for an urgent thoracotomy. Fortunately, blood pressure returned to normal after $1 \mathrm{~h}$. We presumed that blood clots had already obstructed the bleeding point and urgent surgery was not performed. When this multigroove silicone drain was removed, we clearly confirmed that this drain was found to be completely obstructed by some thrombotic clots and soluble gauze for hemostasis called surgicel. The postoperative course was uneventful and this patient was discharged from hospital 5 days later. After 2 weeks, this patient came to the clinic for a follow-up check. Owing to the function of the cardiac and diaphragm de-fibrosis, the blood clot had become free blood that can be easily drained (39). Under the guidance of ultrasound, we used a small 8-F ABLE catheter to drain the blood that gathered in the chest cavity. About 1,400 mL blood in total was drained and this patient was well after 1 year of follow up. Furthermore, we have never experienced complete drain occlusion when using conventional chest drains. Doctors and nurses should always bear in mind that drains may be obstructed regardless of their size and design, and should regularly check that negative pressure is always maintained.

In recent years, some foreign researchers have explored the relationship between postoperative serum CRP level and complications after surgery $(8,9)$. It is commonly believed that CRP is an acute-phase response protein (10). CRP is mainly produced in the liver in response to IL-1 $\beta$ and IL-6 (40). However, there exists some evidence that CRP also has potential anti-inflammatory functions after lung surgery (41). Furthermore, CRP is not only just a simple inflammatory marker but also a component of innate immunity (10). At postoperative day 1 , the amount of serum CRP in the 2 groups further increased $(\mathrm{P}>0.05)$, and the comparison between the 2 groups showed that the levels of serum CRP in the multi-groove silicone drain group at $72 \mathrm{~h}$ after the operation were significantly lower than those in the conventional drain group $(\mathrm{P}<0.05)$. The reasons for high postoperative CRP levels can be varied. The half-life of CRP is about $19 \mathrm{~h}$ and its clearance is independent of its concentration $(41,42)$. Hence, considerable variations in CRP levels are possible within 1 or 2 days. However, pulmonary infectious complications do not play a leading role in postoperative patients, especially in the immediate postoperative stage. The levels of serum CRP in the multigroove silicone drainage group at $72 \mathrm{~h}$ after operation were significantly lower than those in the conventional drain group, showing that the incidence of postoperative complications may be lower in the multi-groove silicone drain group. Nevertheless, we did not obtain a statistically significant difference between the 2 groups $(\mathrm{P}=0.055)$. Therefore, the main reason for the fluctuation in serum CRP level in the postoperative period needs to be further studied.

On the basis of our clinical experience and some data analysis in recent years, multi-groove silicone drains have some advantages in single-port video-assisted thoracoscopic lung cancer surgery. Firstly, this drain can minimize wound tissue trauma and pain around the incision. Secondly, the flexible drains can be placed in a curved position, advancing from the incision to the apex of the chest cavity, and then stretching to the bottom of the chest cavity to attain complete drainage of the chest cavity. Thirdly, this system has greater potential to shorten the length of the chest tube removal time and the average hospital stay time, therefore, the hospital cost can be diminished. However, this drain can be expensive, and we encountered 1 case in which this drain was completely occluded, highlighting some of the challenges of multi-groove silicone drains. So, the multigroove silicone drains could be used in most surgical patients. For those patients with higher susceptibility of postoperative active bleeding such as intraoperative pleural adhesion and coagulation function disorder, we should take cautions. Only if this problem was smoothly solved and multi-groove silicone drains could be used pervasively.

This study has several limitations, including its retrospective nature, and we only used this drain in the lobectomy surgical procedure. Therefore, this drain should also be trialed in other complicated surgeries.

\section{Conclusions}

From this study, the multi-groove silicone drains seem to be effective and can be used as an alternative in most patients. Our findings must be confirmed in a large-sample, prospective randomized controlled study.

\section{Acknowledgments}

Funding: This study was supported by the Shanghai Key Laboratory of Clinical Geriatric Medicine, Shanghai Municipal Key Clinical Specialty (No.: shslczdzk02801), Digital technologies for the postoperative remote care and rehabilitation of thoracic and cardiac surgery patients (No.: 2019YFE0105600). 


\section{Footnote}

Reporting Checklist: The authors have completed the STROBE reporting checklist. Available at https://dx.doi. org/10.21037/jtd-21-1801

Data Sharing Statement: Available at https://dx.doi. org/10.21037/jtd-21-1801

Conflicts of Interest: All authors have completed the ICMJE uniform disclosure form (available at https://dx.doi. org/10.21037/jtd-21-1801). The authors have no conflicts of interest to declare.

Ethical Statement: The authors are accountable for all aspects of the work in ensuring that questions related to the accuracy or integrity of any part of the work are appropriately investigated and resolved. The study was conducted in accordance with the Declaration of Helsinki (as revised in 2013). The study was approved by ethics board of Ningbo No.2 Hospital (No.: 2021-047-01), and individual consent for this retrospective analysis was obtained.

Open Access Statement: This is an Open Access article distributed in accordance with the Creative Commons Attribution-NonCommercial-NoDerivs 4.0 International License (CC BY-NC-ND 4.0), which permits the noncommercial replication and distribution of the article with the strict proviso that no changes or edits are made and the original work is properly cited (including links to both the formal publication through the relevant DOI and the license). See: https://creativecommons.org/licenses/by-nc-nd/4.0/.

\section{References}

1. Sung H, Ferlay J, Siegel RL, et al. Global Cancer Statistics 2020: GLOBOCAN Estimates of Incidence and Mortality Worldwide for 36 Cancers in 185 Countries. CA Cancer J Clin 2021;71:209-49.

2. National Lung Screening Trial Research Team; Aberle DR, Adams AM, et al. Reduced lung-cancer mortality with low-dose computed tomographic screening. N Engl J Med 2011;365:395-409.

3. Madani A, Fiore JF Jr, Wang Y, et al. An enhanced recovery pathway reduces duration of stay and complications after open pulmonary lobectomy. Surgery 2015;158:899-908; discussion 908-10.

4. Bertolaccini L, Brunelli A. Devising the guidelines: the techniques of uniportal video-assisted thoracic surgerypostoperative management and enhanced recovery after surgery. J Thorac Dis 2019;11:S2069-72.

5. Bhandari R, Hao YY. Implementation and Effectiveness of Early Chest Tube Removal during an Enhanced Recovery Programme after Oesophago-gastrectomy. JNMA J Nepal Med Assoc 2015;53:24-7.

6. Ueda K, Haruki T, Murakami J, et al. No Drain After Thoracoscopic Major Lung Resection for Cancer Helps Preserve the Physical Function. Ann Thorac Surg 2019;108:399-404.

7. Wang Z, Lv J, Zhang S, et al. Clinical application of a multi-groove silicone drain combined with unidirectional negative-pressure drainage system in single-operating-port video-assisted thoracoscopic lung cancer surgery: a comparison study. J Int Med Res 2021;49:300060521999766.

8. Miyamura T, Sakamoto N, Kakugawa T, et al. Postoperative acute exacerbation of interstitial pneumonia in pulmonary and non-pulmonary surgery: a retrospective study. Respir Res 2019;20:154.

9. Alcoba G, Keitel K, Maspoli V, et al. A three-step diagnosis of pediatric pneumonia at the emergency department using clinical predictors, C-reactive protein, and pneumococcal PCR. Eur J Pediatr 2017;176:815-24.

10. Pastorino U, Morelli D, Leuzzi G, et al. Baseline and Postoperative C-reactive Protein Levels Predict LongTerm Survival After Lung Metastasectomy. Ann Surg Oncol 2019;26:869-75.

11. Lindenmann J, Fink-Neuboeck N, Taucher V, et al. Prediction of Postoperative Clinical Outcomes in Resected Stage I Non-Small Cell Lung Cancer Focusing on the Preoperative Glasgow Prognostic Score. Cancers (Basel) 2020;12:152.

12. Hu Y, Ren SY, Wang RY, et al. Surgical Outcomes After Neoadjuvant Chemoimmunotherapy for Resectable NonSmall Cell Lung Cancer. Front Oncol 2021;11:684070.

13. Hotta T, Nakashima K, Hata K, et al. High serum C-reactive protein levels predict survival in patients with treated advanced lung adenocarcinoma. J Thorac Dis 2021;13:1476-84.

14. Mendogni P, Mazzucco A, Palleschi A, et al. Uniportal and three-portal video-assisted thoracic surgery pulmonary lobectomy for early-stage lung cancer (UNIT trial): study protocol of a single-center randomized trial. Trials 2021;22:163.

15. Woodfield JC, Sagar PM, Thekkinkattil DK, et al. Accuracy of the Surgeons' Clinical Prediction of 
Postoperative Major Complications Using a Visual Analog Scale. Med Decis Making 2017;37:101-12.

16. He R, Zhu Q, Wang Y, et al. Influence of respiratory function training under the mode of mutual-assisted patients on postoperative pulmonary infection and immune function on lung cancer. Am J Transl Res 2021;13:9260-8.

17. Yan S, Wang X, Wang Y, et al. Intermittent chest tube clamping may shorten chest tube drainage and postoperative hospital stay after lung cancer surgery: a propensity score matching analysis. J Thorac Dis 2017;9:5061-7.

18. Karalapillai D, Weinberg L, Neto A S, et al. Intraoperative ventilator mechanical power as a predictor of postoperative pulmonary complications in surgical patients: A secondary analysis of a randomised clinical trial. Eur J Anaesthesiol 2022;39:67-74.

19. Wei X, Yu H, Dai W, et al. Patient-Reported Outcomes of Video-Assisted Thoracoscopic Surgery Versus Thoracotomy for Locally Advanced Lung Cancer: A Longitudinal Cohort Study. Ann Surg Oncol 2021;28:8358-71.

20. Huang L, Zheng J, Hu R, et al. Uniportal video-assisted thoracoscopic surgery for lung neoplasms with tracheal bronchus: a case report. Transl Cancer Res 2020;9:3721-4.

21. Wu Z, Wang Q, Wu C, et al. Three-port single-intercostal versus multiple-intercostal thoracoscopic lobectomy for the treatment of lung cancer: a propensity-matched analysis. BMC Cancer 2019;19:8.

22. Wang Y, Wang Z, Yao F. The safety and feasibility of three-dimension single-port video-assisted thoracoscopic surgery for the treatment of early-stage lung cancer. J Thorac Dis 2020;12:7257-65.

23. Han D, Cao Y, Wu H, et al. Uniportal video-assisted thoracic surgery for the treatment of lung cancer: a consensus report from Chinese Society for Thoracic and Cardiovascular Surgery (CSTCVS) and Chinese Association of Thoracic Surgeons (CATS). Transl Lung Cancer Res 2020;9:971-87.

24. Borro JM, Regueiro F, Pértega S, et al. Comparative Study of Survival following Videothoracoscopic Lobectomy Procedures for Lung Cancer: Single- versus Multiple-port Approaches. Arch Bronconeumol 2017;53:199-205.

25. Gao Y, Abulimiti A, He D, et al. Comparison of singleand triple-port VATS for lung cancer: A meta-analysis. Open Med (Wars) 2021;16:1228-39.

26. Wu CF, Paradela M, Wu CY, et al. Novice training: The time course for developing competence in single port video-assisted thoracoscopic lobectomy. Medicine
(Baltimore) 2020;99:e19459.

27. Deng B, Qian K, Zhou JH, et al. Optimization of Chest Tube Management to Expedite Rehabilitation of Lung Cancer Patients After Video-Assisted Thoracic Surgery: A Meta-Analysis and Systematic Review. World J Surg 2017;41:2039-45.

28. Nakamura H, Taniguchi Y, Miwa K, et al. The use of Blake drains following general thoracic surgery: is it an acceptable option? Interact Cardiovasc Thorac Surg 2009;8:58-61.

29. Hoeijmakers F, Hartemink KJ, Verhagen AF, et al. Variation in incidence, prevention and treatment of persistent air leak after lung cancer surgery. Eur J Cardiothorac Surg 2021. [Epub ahead of print]. doi: 10.1093/ejcts/ezab376.

30. Ali RK, Kakamad FH, Hama Ali Abdalla S, et al. Management of post lobectomy subcutaneous emphysema; a case report with literature review. Ann Med Surg (Lond) 2021;69:102610.

31. Sakopoulos AG, Hurwitz AS, Suda RW, et al. Efficacy of Blake drains for mediastinal and pleural drainage following cardiac operations. J Card Surg 2005;20:574-7.

32. Gordon BM, Hasaniya NW, Newcombe JB, et al. Blake drains: a novel method of chest drainage after extracardiac fontan operation with autologous pericardium. Ann Thorac Surg 2012;94:1289-94.

33. Chen Y, Wang X, Ding W, et al. Perceptions and Life Experiences of Patients With Wound Healing by Secondary Intention After the Removal of Thoracic Drainage Tube: A Qualitative Study. J Wound Ostomy Continence Nurs 2020;47:124-7.

34. Kouritas VK, Zissis C, Bellenis I. Variation of the postoperative fluid drainage according to the type of lobectomy. Interact Cardiovasc Thorac Surg 2013;16:437-40.

35. Holbek BL, Christensen M, Hansen HJ, et al. The effects of low suction on digital drainage devices after lobectomy using video-assisted thoracoscopic surgery: a randomized controlled trial†. Eur J Cardiothorac Surg 2019;55:673-81.

36. Sakakura N, Fukui T, Mori S, et al. Fluid drainage and air evacuation characteristics of Blake and conventional drains used after pulmonary resection. Ann Thorac Surg 2009;87:1539-45.

37. Saxena P, Kejriwal N, Newman MA. Use of Blake drains following lung resection. Eur J Cardiothorac Surg 2006;30:952; author reply 952-5.

38. Nakamura H, Taniguchi Y, Miwa K, et al. The 19Fr Blake drain versus the $28 \mathrm{Fr}$ conventional drain after a lobectomy 
for lung cancer. Thorac Cardiovasc Surg 2009;57:107-9.

39. Clark G, Licker M, Bertin D, et al. Small size new silastic drains: life-threatening hypovolemic shock after thoracic surgery associated with a non-functioning chest tube. Eur J Cardiothorac Surg 2007;31:566-8.

40. Meyer MM, Brandenburg L, Hudel H, et al. Who Is Afraid of CRP? Elevated Preoperative CRP Levels Might Attenuate the Increase in Inflammatory Parameters in Response to Lung Cancer Surgery. J Clin Med 2020;9:3340.

Cite this article as: Yao Y, Hua Q, Liu S, Yang Z, Shen H, Gao $W$. Efficacy of multi-groove silicone drains in single-port videoassisted thoracoscopic lung cancer surgery and their effect on C-reactive protein: a single-center experience. J Thorac Dis 2021;13(12):6885-6896. doi: 10.21037/jtd-21-1801
41. Mold C, Rodriguez W, Rodic-Polic B, et al. C-reactive protein mediates protection from lipopolysaccharide through interactions with Fc gamma R. J Immunol 2002;169:7019-25.

42. Riedl JM, Barth DA, Brueckl WM, et al. C-Reactive Protein (CRP) Levels in Immune Checkpoint Inhibitor Response and Progression in Advanced Non-Small Cell Lung Cancer: A Bi-Center Study. Cancers (Basel) 2020;12:2319. 